\title{
The effects of Livin shRNA on the response to cisplatin in HepG2 cells
}

\author{
FANGFENG LIU, HONG CHANG, WEI XU and YUNPENG ZHAI
}

Department of General Surgery, Provincial Hospital Affiliated to Shandong University, Jinan, Shandong 250021, P.R. China

Received July 25, 2014; Accepted March 4, 2015

DOI: $10.3892 / 01.2015 .3629$

\begin{abstract}
Hepatocellular carcinoma is a lethal malignancy with poor prognosis, partially due to tumor metastasis, recurrence and resistance to chemo- or radio-therapy. Cisplatin can inhibit cancer cell DNA replication, and is widely used in the clinical treatment of tumors. The present study aimed to generate eukaryotic expression vectors for Livin shRNA and to examine the effects of Livin shRNA on the chemosensitivity of HepG2 hepatocellular carcinoma cells. Eukaryotic expression vectors for Livin shRNA (pSD11-U6/Neo/GFP/Livin) were designed and constructed. The HepG2 hepatocellular carcinoma cell line was transfected with this vector using the liposome method. The expression levels of Livin mRNA and protein were measured by quantitative polymerase chain reaction and western blot analysis. The rate of cell growth inhibition was measured using MTT assay following treatment of the cells with cisplatin $(2.0 \mathrm{mg} / \mathrm{l})$. DNA sequencing confirmed that the construction of the eukaryotic expression vector for Livin shRNA had been successful. Transfection of these vectors into HepG2 cells led to a significant reduction in the expression levels of Livin mRNA and protein $(\mathrm{P}<0.05)$. Cisplatin treatment was associated with significantly higher rates of cell growth inhibition in HepG2 cells transfected with Livin shRNA compared with those that were not transfected $(\mathrm{P}<0.05)$. The vectors constructed in the present study produced effective inhibition of the Livin gene in HepG2 cells and increased the chemosensitivity of hepatocellular carcinoma cells.
\end{abstract}

\section{Introduction}

Hepatocellular carcinoma is a common malignant tumor with poor prognosis. RNA interference (RNAi) can reduce oncogene expression and alter the biology of tumor cells.

Correspondence to: Professor Hong Chang, Department of General Surgery, Provincial Hospital Affiliated to Shandong University, 324 Jing-5-Wei-7 Road, Jinan, Shandong 250021, P.R. China

E-mail: hchang0721@163.com

Key words: Livin, RNA interference, hepatocellular carcinoma
Therefore, application of RNAi to enhance the chemosensitivity of hepatocellular carcinoma to cisplatin, a commonly used anticancer drug that inhibits cancer cell DNA replication, is a potentially important therapeutic strategy (1).

Livin is a member of the inhibitor of the apoptosis (IAP) family and the baculoviral IAP repeat-containing 7 (BIRC7) gene that encodes Livin is located on human chromosome $20 \mathrm{q} 13.3$, is $46 \mathrm{~kb}$ in length and comprises 7 exons and 6 introns (2). Overexpression of Livin has been observed in certain types of cancer tissues and cell lines (3). This may inhibit the cellular apoptosis induced by several anticancer agents. It is also associated with drug resistance in cancer cells $(2,4,5)$. RNA interference (RNAi) is the phenomenon by which double-stranded RNA molecules homologous to a target gene are processed by the enzyme Dicer to produce active small interfering RNA (siRNA) molecules. siRNAs form a complex called the RNA-induced silencing complex (RISC) that includes endonucleases termed argonaut proteins. The RISC, guided by the siRNA, binds to and specifically cleaves mRNA from the target gene, leading to its degradation. This is one form of post-translational gene silencing (2).

In the present study, a eukaryotic expression vector for Livin was constructed. The use of short hairpin (sh)RNA vectors is a popular approach for delivering siRNA. Using the RNAi approach, Livin expression in the HepG2 hepatocellular carcinoma cells was inhibited and the effects of this on the chemosensitivity of the cells were investigated.

\section{Materials and methods}

Primary reagents. Fetal bovine serum (FBS), Dulbecco's modified Eagle's medium (DMEM), Opti-MEM, and a Lipofectamine 2000 transfection kit were purchased from Invitrogen Life Technologies (Carlsbad, CA, USA). RNAiso Plus, a PrimeScript ${ }^{\mathrm{TM}}$ Reverse Transcription (RT) Reagent Kit with gDNA Eraser, and an LA PCR Amplification Kit Version 2.1 were purchased from Takara Biotechnology Co., Ltd. (Dalian, China). Radioimmunoprecipitation assay (RIPA) buffer, phenylmethylsulfonyl fluoride (PMSF) protease inhibitor and a BCA-100 protein assay kit were purchased from Genechem Co., Ltd. (Shanghai, China). Monoclonal mouse anti-human antibodies against Livin (catalog no. ab118026) and GAPDH (catalog no. ab8245) were purchased from Abcam (Shanghai, China). Horseradish peroxidase (HRP)-labeled goat anti-mouse IgG antibody 


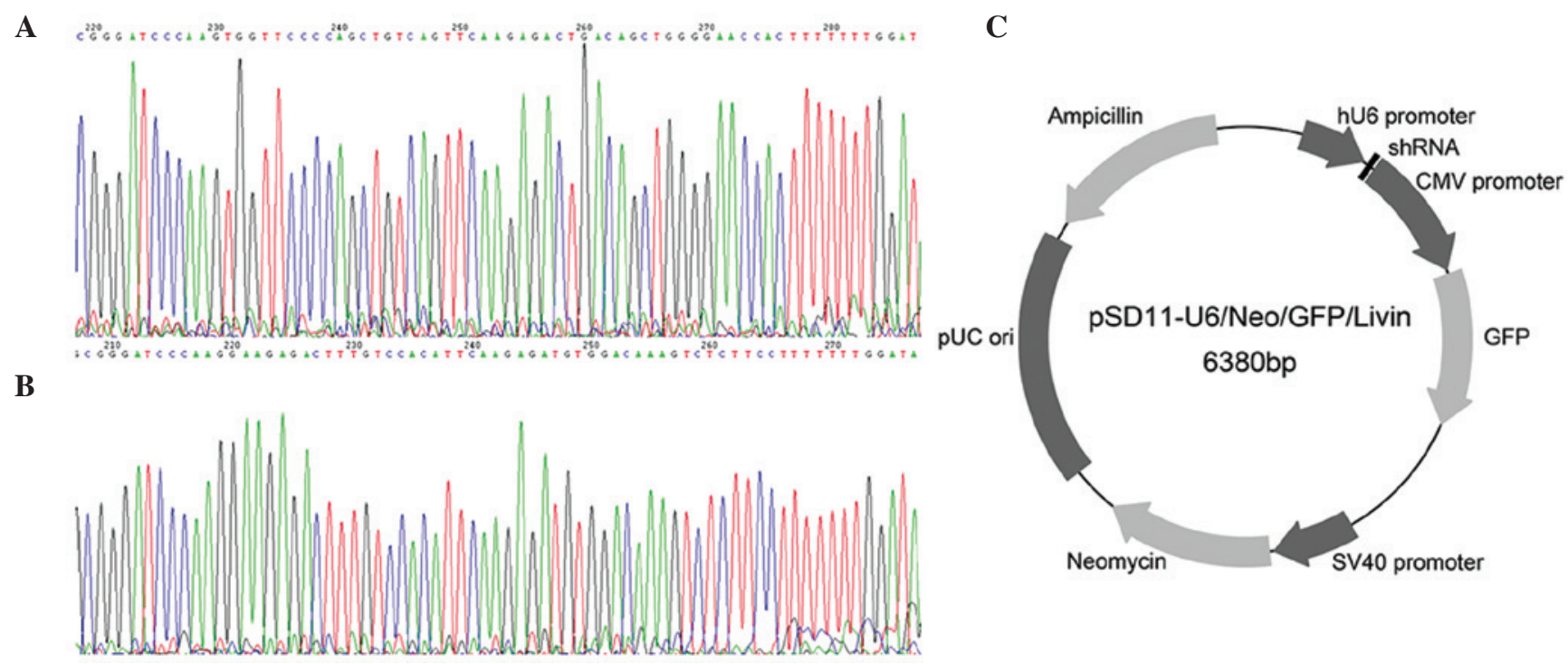

Figure 1. Sequencing of recombinant plasmids. (A) Livin 1 and (B) Livin 2. (C) pSD11-U6/Neo/GFP/Livin vector structure. Structure of recombinant plasmid pSD11 (6380 bp). Vector information (in bp): hU6 promoter, 289-616; Polylinker, 617-640; CMV promoter, 671-1259; GFP, 1283-2002; SV40 promoter, 2672-3049; Neomycin, 3077-3871; pUC ori, 4108-5276; Ampicillin, 6233-5373. bp, base pairs.

(catalog no. IH-0041) was purchased from Beijing Dingguo Changsheng Co., Ltd. (Beijing, China). MTT was purchased from Sigma-Aldrich (St. Louis, MO, USA).

Cell culture. The human hepatocellular carcinoma cell line HepG2 was obtained from the central laboratory of Provincial Hospital Affiliated to Shandong University (Shandong, China). HepG2 cells were seeded at a density of $4 \times 10^{5}$ cells/ well in 6-well plates with DMEM containing 10\% FBS in an incubator at $37^{\circ} \mathrm{C}$ and $5 \% \mathrm{CO}_{2}$. These cells became adherent and $0.25 \%$ Trysin-EDTA (Gibco Life Technologies, Carlsbad, CA, USA) was used for passage.

Construction of the Livin shRNA vector. The full-length mRNA sequence of the Livin gene (BIRC7; NCBI gene ID, 79444) was identified in the NCBI gene database. Based on shRNA design principles and relevant literature (6), two 19-nucleotide (nt) sequences were selected for use as target sequences for the siRNA vector construction as follows: Livin 1, 5'-GTGGTTCCCCAGCTGTCAG-3' (611-629) and Livin 2, 5'-GGAAGAGACTTTGTCCACA-3' (648-666). BLAST analysis confirmed that the selected sequences were not homologous to other human gene sequences. The sequences were flanked by BamHI and HindIII restriction sites at each end. Sense and antisense strands were connected by a 9-nt loop sequence (TTCAAGAGA). Clone construction involved direct annealing ligation; the shRNA coding fragments flanked by restriction sites were annealed and then directly ligated (T4 DNA Ligase; Invitrogen Life Technologies) with the vector prepared by restriction digestion. The structure of the recombinant plasmid pSD11-U6/Neo/ GFP/Livin is presented in Fig. 1. The 2 expression vectors produced in the present study are designated pSD11-Livin1 and pSD11-Livin2. A negative control vector, termed pSD11-NC, was also constructed. It was constructed by re-ligation following the restriction endonuclease digestion. The construction of the Livin shRNA vector was performed at Shanghai Jikai Biotechnology Co., Ltd. (Shanghai, China) and sequencing was performed at Sangon Biotech Co., Ltd. (Shanghai, China).

Cell transfection. At $24 \mathrm{~h}$ prior to transfection, the HepG2 cells were trypsinized ( $0.25 \%$ trypsin in DMEM) and seeded in 6 -well plates at a density of $2 \times 10^{5}$ cells/well. The cells were cultured in DMEM for $24 \mathrm{~h}$, reaching $80 \%$ confluence, which was confirmed by inverted microscopy (CKX31 Inverted Microscope, Olympus Corporation, Tokyo, Japan). Cell transfection was performed in accordance with the manufacturer's instructions using the Lipfectamine 2000 kit. The experiments were performed in 4 groups as follows: The Livin 1 group (cells transfected with pSD11-Livin1); the Livin 2 group (cells transfected with pSD11-Livin2); the $\mathrm{NC}$ group (cells transfected with pSD11-NC); and the control group (untransfected cells).

Reverse transcription-quantitative polymerase chain reaction $(R T-q P C R)$. At $48 \mathrm{~h}$ following transfection, the total RNA was extracted from cells in each group. RT was performed using the RNAiso Plus kit according to the manufacturer's instructions. The reaction conditions were as follows: $37^{\circ} \mathrm{C}$ for $15 \mathrm{~min}$ and $85^{\circ} \mathrm{C}$ for $5 \mathrm{sec}$ and then the mix was stored at $4^{\circ} \mathrm{C}$. Primer Premier software (version 5.0; http://www.premierbiosoft.com/ primerdesign/index.html) was used to design primers for the fluorescent qPCR. The sequences of the primers used were as follows: Livin, F 5'-CCATCAGCCCCCATTTCT-3' and R 5'-CCATCAGCCCCCATTTCT-3' (the amplicon was 79 bp in length); GAPDH, F 5'-TGCACCACCAACTGCTTAGCA-3' and R 5'-TGCACCACCAACTGCTTAGCA-3' (the amplicon was 87 bp in length). The primers were synthesized by Shanghai Sangon Biotech Co., Ltd. The qPCR was performed on a LightCycler 480 Instrument II using SYBR Green I (Roche Diagnostics, Shanghai, China). The thermal cycling conditions were as follows: Pre-denaturation at $95^{\circ} \mathrm{C}$ for $30 \mathrm{sec}$ followed by 40 cycles of amplification at $95^{\circ} \mathrm{C}$ for $5 \mathrm{sec}$ and $60^{\circ} \mathrm{C}$ for $20 \mathrm{sec}$. A melting 
curve analysis was performed following PCR. To exclude false positive results, PCR for the Livin gene and GAPDH internal reference gene were conducted using blank controls to which no cDNA had been added.

Western blot analysis. At $72 \mathrm{~h}$ following transfection, cells were collected from each group. RIPA lysis buffer and PMSF (at a ratio of 100:1) were added to the cell pellets and incubated in an ice bath for $30 \mathrm{~min}$. The samples were centrifuged at 2,000 x g for $30 \mathrm{~min}$ at $4^{\circ} \mathrm{C}$. The supernatant was collected and mixed with protein loading buffer (Shenergy Biocolor Bioscience \& Technology Company, Shanghai, China) and incubated at $100^{\circ} \mathrm{C}$ for $10 \mathrm{~min}$. Protein samples were resolved by $10 \%$ polyacrylamide gel electrophoresis and then transferred to a polyvinylidene fluoride membrane (Merck Millipore, Darmstadt, Germany). The membranes were then blocked with Tris-buffered saline and $0.05 \%$ Tween-20 (TBS-T) containing $5 \%$ skimmed milk powder at $37^{\circ} \mathrm{C}$ for $60 \mathrm{~min}$. The mouse anti-human Livin (dilution, 1:1,000) and GAPDH (dilution, 1:3,000) antibodies were added and incubated at $4^{\circ} \mathrm{C}$ overnight. The membranes were then washed with TBS-T and incubated with the HRP-labeled goat anti-mouse $\operatorname{IgG}$ antibody (dilution, 1:3,000) with shaking at room temperature for $1 \mathrm{~h}$. Following thorough washing with TBS-T, the membranes were immersed in a solution of enhanced chemiluminescence liquid (Immobilon Western Chemiluminescent HRP Substrate, Merck Millipore) and incubated for $5 \mathrm{~min}$ at room temperature. The images were visualized using gel imaging equipment; bands from the electrophoresis were analyzed with an AlphaImager ${ }^{\circledR} 2200$ image analyzer (ProteinSimple Bioscience \& Technology Co., Ltd., Shanghai, China).

MTT assay. One day prior to transfection, HepG2 cells were seeded in 96 -well plates at a density of $5 \times 10^{5}$ cells/well. Grouping was as described above and each group consisted of 5 replicates. In addition, a blank group was set up containing HepG 2 cells only and no added reagents. The transfection procedure was performed using the Lipofectamine $2000 \mathrm{kit}$, according to the manufacturer's instructions. At $48 \mathrm{~h}$ following transfection, DMEM containing 10\% FBS and $2.0 \mathrm{mg} / \mathrm{l} \mathrm{cispl-}$ atin (central laboratory of Provincial Hospital Affiliated to Shandong University) was added to the cells. The cells were cultured for an additional 24, 36 or $48 \mathrm{~h}$. The culture medium was removed and replaced with $20 \mu 1 \mathrm{MTT}(5 \mathrm{mg} / \mathrm{ml})$ solution at the time points indicated. The cells were incubated at $37^{\circ} \mathrm{C}$ with $5 \% \mathrm{CO}_{2}$ for $4 \mathrm{~h}$ and then $150 \mu \mathrm{l}$ dimethyl sulfoxide was added to each well and oscillated for $10 \mathrm{~min}$. The optical density (OD) was measured using a Microplate Reader (DNM-9602; Nanjing Perlove Eadial-Video Equipment Co., Ltd., Nanjing, China) at $490 \mathrm{~nm}$. The rate of cell growth inhibition (IR) was calculated using the following formula: $\mathrm{IR}=\left(1-\mathrm{OD}_{\text {Experimental }} / \mathrm{OD}_{\text {Blank }}\right) \times 100$.

Statistical analysis. SPSS statistical software, version 15.0 (SPSS, Inc., Chicago, IL, USA) was used for analysis. Data are expressed as the mean \pm standard error. The statistical significance was evaluated by a one-way analysis of variance with Tukey's test. $\mathrm{P}<0.05$ was considered to indicate a statistically significant difference.

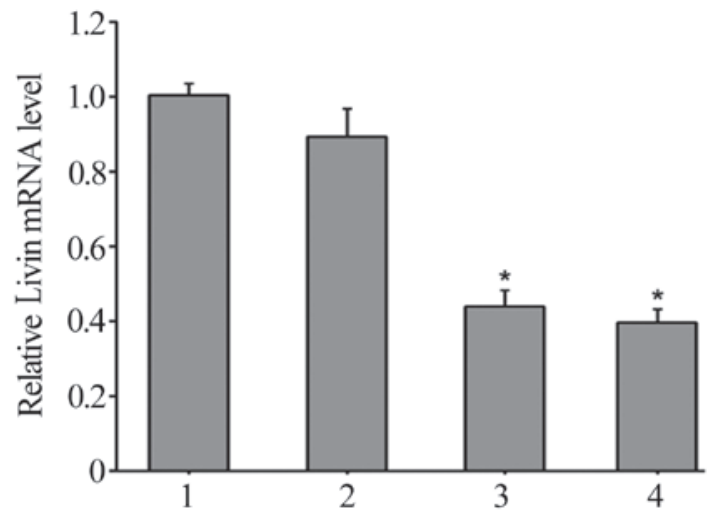

Figure 2. The relative Livin mRNA expression in HepG2 cells following transfection with Livin shRNA expression vectors, was quantified by RT-qPCR. 1, control group (untransfected cells); 2, NC group (cells transfected with pSD11-NC); 3, Livin 1 group (cells transfected with pSD11-Livin1); 4, Livin 2 group (cells transfected with pSD11-Livin2). ${ }^{*} \mathrm{P}<0.05$ vs. the $\mathrm{NC}$ and control groups.

\section{Results}

Verification of construction of recombinant plasmid. DNA sequencing confirmed that the shRNA coding sequences were correctly inserted into the plasmid vector pSD11-U6/Neo/GFP and that recombinant plasmids pSD-11-Livin1 and pSD11-Livin2 were as designed. Thus, the recombinant plasmids were successfully prepared.

HepG2 cellular mRNA expression changes following transfection. RT-qPCR was performed to quantify the changes in Livin mRNA expression levels. The melting curve for the Livin PCR product peaked at $89.9^{\circ} \mathrm{C}$, and the melting curve for the GAPDH PCR product peaked at $88.1^{\circ} \mathrm{C}$. The PCR products produced a single peak, excluding the possibility of non-specific amplification. The negative control group without cDNA did not produce a melting curve, excluding the possibility of false positive results. GAPDH served as an internal reference gene and relative quantification (Livak method) (7) was used to calculate the relative expression level, presented as $\Delta \Delta \mathrm{Ct}$. The results demonstrated that $48 \mathrm{~h}$ following transfection, the relative levels of Livin mRNA expression in HepG2 cells in the Livin 1, Livin 2, NC and control groups were $0.44 \pm 0.04,0.40 \pm 0.03,0.90 \pm 0.07$ and $1.00 \pm 0.03$, respectively (Fig. 2). The expression levels in the Livin 1 and Livin 2 groups were significantly lower than those of the $\mathrm{NC}$ and control groups $(\mathrm{P}<0.05)$. These results indicated that pSD11-Livin1 and pSD-11-Livin2 transfection in HepG2 cells specifically reduced the expression levels of Livin mRNA (Fig. 2).

Changes in Livin protein expression in HepG2 cells following transfection. Bands from the western blots were analyzed using an Alpha Imager 2200. The results of this analysis demonstrated that $72 \mathrm{~h}$ following transfection, the relative expression levels of Livin protein in the HepG2 cells in the Livin 1, Livin 2 and NC groups were $0.53 \pm 0.03$, $0.46 \pm 0.07$, and $0.90 \pm 0.06$, respectively relative to the control group, which was set to 1 . There were significantly lower protein levels in the Livin 1 and Livin 2 groups compared 
Table I. Rate of cell growth inhibition (\%) by cisplatin in HepG2 cells following transfection with Livin shRNA expression vectors at different time points.

Percentage cell growth inhibition at different time points

\begin{tabular}{lccc}
\cline { 2 - 4 } Group & $24 \mathrm{~h}$ & $36 \mathrm{~h}$ & $48 \mathrm{~h}$ \\
\hline Control & $6.12 \pm 0.88$ & $14.75 \pm 1.10$ & $23.03 \pm 1.07$ \\
NC & $6.51 \pm 1.03$ & $15.34 \pm 1.11$ & $23.59 \pm 1.32$ \\
Livin 1 & $15.87 \pm 0.84^{\mathrm{a}}$ & $28.00 \pm 0.85^{\mathrm{a}}$ & $36.76 \pm 1.20^{\mathrm{a}}$ \\
Livin 2 & $16.57 \pm 1.03^{\mathrm{a}}$ & $28.10 \pm 0.96^{\mathrm{a}}$ & $37.10 \pm 1.48^{\mathrm{a}}$
\end{tabular}

${ }^{\mathrm{a}} \mathrm{P}<0.05$ vs. the $\mathrm{NC}$ and control groups. Control, untransfected cells; $\mathrm{NC}$, cells transfected with pSD11-NC; Livin 1, cells transfected with pSD11-Livin1; Livin 2, cells transfected with pSD11-Livin2.
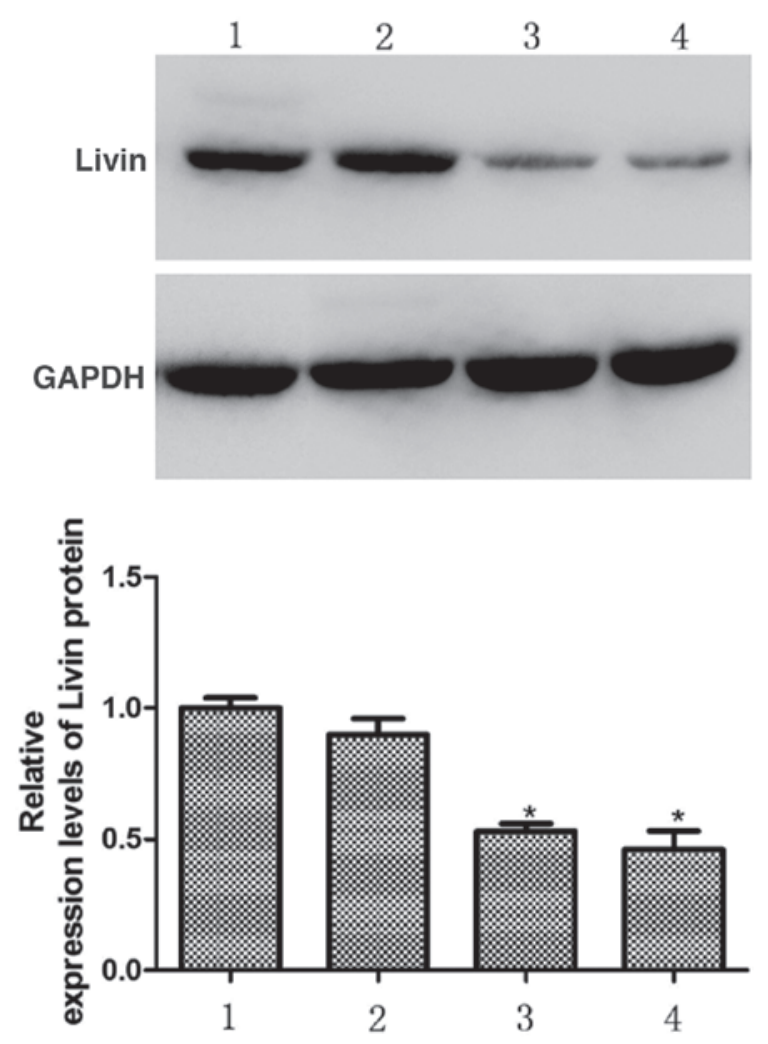

Figure 3. The relative Livin protein expression in HepG2 cells following transfection with Livin shRNA expression vectors was examined by western blot analysis. 1, control group (untransfected cells); 2, NC group (cells transfected with pSD11-NC); 3, Livin 1 group (cells transfected with pSD11-Livin1); 4 , Livin 2 group (cells transfected with pSD11-Livin2). ${ }^{*} \mathrm{P}<0.05$ vs. NC and control groups.

with the $\mathrm{NC}$ and control groups $(\mathrm{P}<0.05)$. These results indicate that pSD-11-Livin1 and pSD11-Livin2 transfection in HepG2 cells specifically downregulates the expression of Livin protein (Fig. 3).

Inhibition of HepG2 cell growth by cisplatin. The results of the MTT assay demonstrated that at a cisplatin concentration of $2 \mathrm{mg} / \mathrm{l}$, each group exhibited various degrees of growth inhibition. This was recorded at 24,36 and $48 \mathrm{~h}$, and the rate of inhibition gradually increased over time. The rates of cell growth inhibition in the Livin 1 and Livin 2 groups were significantly higher than in the $\mathrm{NC}$ and control groups $(\mathrm{P}<0.05$; Table I). No statistically significant differences were identified between the Livin 1 and Livin 2 groups, or between the NC and control groups (Table I).

\section{Discussion}

Hepatocellular carcinoma is a common type of malignant tumor in gastroenterological clinical practice. The efficacy of traditional therapeutics for hepatocellular carcinoma, including surgical resection and chemotherapy, is often limited and the disease is difficult to cure; this may be related to the presence of chronic liver conditions, such as hepatitis and cirrhosis. The relationship between changes in gene expression in cancer cells and their chemosensitivity has become an important part of anticancer research $(8,9)$. RNAi provides considerable potential in this line of research. Ashhab et al (10) described 6 approaches for introducing siRNAs into mammalian cells. Of these approaches, the design and introduction of shRNA expression vectors into mammalian cells that produce siRNA against a target gene allows relatively stable and specific RNAi without any of the effects of directly introducing siRNA into cells. This strategy also permits inhibition of multiple variants of a single gene or a sequence shared among multiple genes (11).

The post-transcriptional processing of the Livin gene forms 2 types of mature Livin mRNA, Livin $\alpha$ and Livin $\beta$, which contain 1,351 and 1,297 nts, respectively. The difference between these 2 types of mRNA is a fragment of $54 \mathrm{nts}$; the rest of the sequence is the same. Livin $\alpha$ and $\beta$ encode a protein with the required structure for anti-apoptotic function, a baculovirus IAP repeat (BIR) domain. Although the 2 proteins differ in 18 amino acid residues in this domain, their functions are essentially similar; to bind caspase via the BIR domain, inhibiting its activity and thus inhibiting cancer cell mitochondrial apoptosis $(12,13)$. A number of studies have demonstrated that the overexpression of Livin in certain types of cancer tissues and cell lines can inhibit the apoptosis induced by multiple anticancer agents $(14,15)$. Other previous studies have demonstrated Livin overexpression to be associated with drug resistance in cancer cells (16-18). Previous studies have also produced shRNA expression vectors and used them to stably silence the Livin gene in human bladder cancer T24 cells (19), HeLa cervical cancer (20) and Lovo colon cancer (3) cell lines. The impact of shRNA expression vectors on cancer cell 
growth and apoptosis has also been studied. Liang et al (21) demonstrated that Livin expression in HepG2 cells increased significantly following the addition of a chemotherapeutic agent, contributing to resistance to chemotherapy. To the best of our knowledge, there have not yet been any studies on the use of RNAi to reduce Livin expression in HepG2 hepatocellular carcinoma cells, or the effects on chemosensitivity.

In the present study, 2 target sequences were selected from the sequence shared by Livin $\alpha$ and $\beta$ mRNAs, and were used to construct shRNA eukaryotic vectors. Following transfection of the hepatocellular carcinoma cell line HepG2 with these vectors, the expression levels of Livin mRNA and protein were evaluated with RT-qPCR and western blot analysis. The results demonstrated that Livin expression levels were downregulated in HepG2 cells transfected with pSD11-Livin1 and pSD-11-Livin2. The differences from cells transfected with pSD11-NC and control cells were statistically significant, indicating the effectiveness of the selected target sequences. Further experiments were performed on HepG2 cells $48 \mathrm{~h}$ following Livin transfection. These cells were treated with $2.0 \mathrm{mg} / \mathrm{l}$ cisplatin for various lengths of time and then an MTT assay was performed to determine the absorbance (OD values) and to calculate the rates of cell growth inhibition. The results demonstrated that the cell growth inhibition rate attributable to cisplatin in HepG2 cells transfected with pSD11-Livin1 and pSD11-Livin2 were significantly higher than those in cells transfected with pSD11-NC and untransfected control cells. The present study also indicated that transfection with an shRNA eukaryotic expression vector against Livin mRNA may inhibit Livin gene expression and effectively increase the chemosensitivity of hepatocellular carcinoma cells. The present study provides novel experimental evidence that manipulating the expression of the Livin gene in hepatocellular carcinoma cells can affect their chemosensitivity.

It has been previously demonstrated that chemotherapeutic agents affect the changes made by Livin gene silencing on apoptotic pathways in cancer cells $(21,22)$. Crnkovic-Mertens et al (6) demonstrated that in HeLa cells treated with Livin shRNA and a reduced dose of chemotherapeutic agent, Livin gene expression reduced and caspase-3 expression increased significantly. Quintieri et al (23) stated that the activation of caspases is key to the cancer cell apoptosis that is induced by chemotherapeutic agents, and that the reduction in caspase activity caused by the factors in cancer cells is a notable cause of the reduction in their chemosensitivity. In the present study, Livin gene expression was increased in hepatocellular carcinoma cells, which may alter the apoptotic pathways, leading to the inhibition of apoptosis induced by chemotherapeutic agents. The combined use of chemotherapeutic agents and RNAi technology to investigate the changes in apoptotic pathways in cancer cells warrants further study. The potential results may aid in the improvement of canonical chemotherapeutic agents and the development of novel adjuvant anticancer drugs.

\section{Acknowledgements}

The present study was supported by the Natural Science Foundation of Shandong province (grant no. Y2008C110, awarded to Professor Hong Chang).

\section{References}

1. Sibley CR, Seow Y and Wood MJ: Novel RNA-based strategies for therapeutic gene silencing. Mol Ther 18: 466-476, 2010.

2. Chen X, Wang T, Yang D, et al: Expression of thr IAP protein family acts cooperatively to predict prognosis in human bladder cancer patients. Oncol Lett 5: 1278-1284, 2013.

3. Liu X, Wang A, Gao H, et al: Expression and role of the inhibitor of apoptosis protein livin in chemotherapy sensitivity of ovarian carcinoma. Int J Oncol 41: 1021-1028, 2012.

4. Wang X, Xu J, Ju S, et al: Livin gene plays a role in drug resistance of colon cancer cells. Clin Biochem 43: 655-660, 2010.

5. Dubrez-Daloz L, Dupoux A and Cartier J: IAPs: more than just inhibitors of apoptosis proteins. Cell Cycle 7: 1036-1046, 2008.

6. Crnkovic-Mertens I, Hoppe-Seyler F and Butz K: Induction of apoptosis in tumor cells by siRNA-mediated silencing of the livin/ML-IAP/KIAP gene. Oncogene 22: 8330-8336, 2003.

7. Schmittgen TD and Livak KJ: Analyzing real-time PCR data by the comparative C(T) method. Nat Protoc 3: 1101-1108, 2008.

8. Kempkensteffen C, Hinz S, Krause H, et al: Expression of splicing variants of the inhibitor of apoptosis livin in testicular germ cell tumors. Tumour Biol 29: 76-82, 2008.

9. Augello C, Caruso L, Maggioni M, et al: Inhibitors of apoptosis proteins (IAPs) expression and their prognostic significance in hepatocellular carcinoma. BMC Cancer 9: 125, 2009.

10. Ashhab Y, Alian A, Polliack A, et al: Two splicing variants of a new inhibitor of apoptosis gene with different biological properties and tissue distribution pattern. FEBS Lett 495: 56-60, 2001.

11. Brummelkamp TR, Bernards R and Agami R: A system for stable expression of short interfering RNAs in mammalian cells. Science 296: 550-553, 2002.

12. Takeuchi H, Kim J, Fujimoto A, et al: X-Linked inhibitor of apoptosis protein expression level in colorectal cancer is regulated by hepatocyte growth factor/C-met pathway via Akt signaling. Clin Cancer Res 11: 7621-7628, 2005.

13. Yuan D, Liu L, Xu H and Gu D: The effects on cell growth and chemosensitivity by livin RNAi in non-small cell lung cancer. Mol Cell Biochem 320: 133-140, 2009.

14. Wang R, Lin F, Wang X, et al: Silencing Livin gene expression to inhibit proliferation and enhance chemosensitivity in tumor cells. Cancer Gene Ther 15: 402-412, 2008.

15. Xu M, Xia LP, Fan LJ, et al: Livin and caspase-3 expression are negatively correlated in cervical squamous cell cancer. Eur J Gynaecol Oncol 34: 152-155, 2013.

16. Myung DS, Park YL, Chung CY, et al: Expression of Livin in colorectal cancer and its relationship to tumor cell behavior and prognosis. PLoS One 8: e73262, 2013.

17. Li F, Yin X, Luo X, et al: Livin promotes progression of breast cancer through induction of epithelial-mesenchymal transition and activation of AKT signaling. Cell Signal 25: 1413-1422, 2013.

18. Li X, Fan S, Li L, et al: RNA interference-mediated knockdown of Livin suppresses cell proliferation and invasion and enhances the chemosensitivity to cisplatin in human osteosarcoma cells. Int J Oncol 43: 159-168, 2013.

19. Yang D, Song X, Zhang J, et al: Suppression of livin gene expression by siRNA leads to growth inhibition and apoptosis induction in human bladder cancer T24 cells. Biosci Biotechnol Biochem 74: 1039-1044, 2010.

20. Yu L and Wang Z: Effects of Livin gene RNA interference on apoptosis of cervical cancer HeLa cells and enhanced sensitivity to cisplatin. J Huazhong Univ Sci Technolog Med Sci 29: 625-630, 2009.

21. Liang SR, Hu GR, Fang LJ, et al: CpG oligodeoxynucleotides enhance chemosensitivity of 5-fluorouracil in HepG2 human hepatoma cells via downregulation of the antiapoptotic factors survivin and livin. Cancer Cell Int 13: 106, 2013.

22. Choi J, Hwang YK, Sung KW, et al: Expression of Livin, an antiapoptotic protein, is an independent favorable prognostic factor in childhood acute lymphoblastic leukemia. Blood 109: 471-477, 2007.

23. Quintieri L, Fantin M and Vizler C: Identification of molecular determinants of tumor sensitivity and resistance to anticancer drugs. Adv Exp Med Biol 593: 95-104, 2007. 